\title{
Prognostic Biomarker MUC4 Mutations in Colon Adenocarcinoma Correlating with Tumor Microenvironment
}

\section{Ting Li}

Xinjiang Medical University

\section{Wenjia Hui}

People's Hospital of Xinjiang Uygur Autonomous Region

Feng Gao ( $\nabla$ xjgf@sina.com )

Xinjiang Medical University

\section{Research Article}

Keywords: colon adenocarcinoma $₫$ MUC4『tumor mutation burden, tumor microenvironment, prognosis

Posted Date: January 7th, 2021

DOl: https://doi.org/10.21203/rs.3.rs-137615/v1

License: (c) (i) This work is licensed under a Creative Commons Attribution 4.0 International License.

Read Full License 


\section{Abstract}

Background: Immunotherapy is a new strategy for Colorectal cancer (CRC) treatment. Tumor mutation burden (TMB) may act as an emerging biomarker for predicting responses to immunotherapy. Nevertheless, no studies investigate if these gene mutations correlate to TMB and tumor-infiltrating immune cells.

Methods: Somatic mutation data for CRC samples were obtained from The Cancer Genome Atlas (TCGA) and the International Cancer Genome Consortium (ICGC) datasets. Then, we investigated the relationship between these mutant genes, TMB and overall survival outcomes. GSEA analysis was performed to explore the underlying mechanism of mutant gene. Finally, we further verified the connection between gene mutations and immune response.

Results: We identified 17 common mutant genes shared by both two cohorts. Further analysis found that MUC4 mutation was strongly related to higher TMB and predicted a poorer prognosis. Moreover, functional enrichment analysis of samples with MUC4 mutation revealed that they were involved in regulating the natural killer cell mediated cytotoxicity signaling pathway. Significant changes in the proportion of the immune cells of $\mathrm{CD}^{+} \mathrm{T}$ cells, activated NK cells, M1 macrophages and resting memory $\mathrm{CD} 4^{+} \mathrm{T}$ cells were observed using the CIBERSORT algorithm.

Conclusions: Our research revealed that MUC4 mutation significantly correlated with increased TMB, a worse prognosis and modulating the immune microenvironment, which may be considered a biomarker to predict the outcome of the immune response in colorectal cancer.

\section{Background}

Colon adenocarcinoma (COAD) is one of the most aggressive malignancies. It is also a heterogeneous disease at the molecular level resulting from complex genetic and epigenetic alterations[1]. The incidence of COAD tends to increase year by year in China[2], Though the development at full speed of treatments, such as targeted therapies, chemotherapy, surgery and radiotherapy, high recurrence rate and poor prognosis remain a concern[3].

Breakthroughs have been made in immunotherapy for COAD. It is considered to be a promising treatment with great potential in the future, particularly for high microsatellite instability (MSI-H) or deficient mismatch repair (MMR). Researchers have recently found that the tumor mutation burden (TMB) is an emerging characteristic of cancer for assessing the effectiveness of immunotherapy[4,5]. Tumor cells with mutations might activate $T$ cells, causing tumor cells to be attacked and cleared by immune system[6, 7]. Patients with high TMB were highly sensitive to immunotherapy according to several previous studies[4, 8]. At present, several clinical studies have confirmed that TMB had prognostic value for COAD immunotherapy[9-11]. Increased mutation load is associated with microsatellite instability[12]. Another study result suggests that TMB has a certain correlation with chemotherapy results in COAD 
patients. Therefore, TMB potential to act as sensitive biomarkers or predicting immunotherapy response. Nevertheless, the immune landscape between tumors with different TMB was unclear in COAD patients.

At present, we explored the somatic mutations of COAD patients in TCGA and ICGC data portal. The common mutant genes shared by these two cohorts were identified. Then, we investigated the relationship between these mutant genes, TMB and overall survival outcomes. Finally, we further analyzed the connection between gene mutations and tumor microenvironment. Overall, our study aimed to provides additional prognostic information for immunotherapy in colon adenocarcinoma.

\section{Methods}

\section{Data source}

The mutation data were downloaded from the TCGA (https://tcga- data.nci.nih.gov/tcga/) and ICGC dataset (https://dcc.icgc.org/). The TCGA dataset including 510 American COAD samples, 434 patients had complete clinical information. The ICGC portal included 321 Chinese COAD samples.

\section{TMB value estimation}

TMB was referred to the average number of genes mutations per megabase (Mb). TSV files, including data of somatic variants for Chinese COAD samples, were annotated and visualized by the GenVisR package. The project "TCGA-COAD" including data of somatic variants for American COAD samples, which was downloaded as a MAF file that was detected using VarScan and visualized with the GenVisR package. The TMB estimate for each sample is equal to the total number of mutation frequency/38. The $38 \mathrm{Mb}$ was routinely used to estimate the exome size of a human.

\section{Gene set enrichment analysis}

COAD patients from the TCGA dataset were split into two groups based on the mutation status of MUC4. c2.cp.kegg.v7.2.symbols.gmt as the reference gene sets using GSEA software (4.0.1), The cut-off criterion was $\mathrm{P}<0.05$.

\section{Estimate of immune cells infiltration}

CIBERSORT is a deconvolution tool that can analyze immune cell landscapes[13]. It was performed to quantify the fractions of immune cells at a threshold of P-value $<0.05$. The result of immune cells distribution was visualized by "vioplot" package.

\section{Statistical analysis}


"Survminer" R package was used to draw the survival curve. Kaplan-Meier(KM) method and log-rank test were performed to compare the survival outcomes. Univariate and multivariate Cox regression was utilized to investigated if the MUC4 mutation was an independent prognostic parameter. Statistical analyses were performed with $\mathrm{R}$ software (version 4.0.0). P-value $<0.05$ was considered significant.

\section{Results}

\section{The mutation spectrum in COAD}

The waterfall diagram revealed the 30 genes with high mutation rate from the TCGA cohort (Figure 1a). At the same time, we explored 30 frequently mutated genes from the ICGC dataset (Figure 1b). The results showed that some genes frequently mutated in both two cohorts. Thus, the Venn diagram visualizes the overlapping genes, including APC, TP53, TTN, KRAS, MUC16, MUC4, SYNE1, FLG, FAT4, OBSCN, FAT3, RYR2, PIK3CA, FBXW7, DNAH11, MUC5B and ZFHX4 (Figure 1C).

\section{MUC4 mutation is linked with high TMB and inferior prognosis in COAD patients}

The result revealed that mutation in TTN, MUC16, MUC4, SYNE1, FLG, FAT4, OBSCN, FAT3, RYR2, PIK3CA, FBXW7, DNAH11, MUC5B and ZFHX4 presented relatively higher TMB in the COAD samples (Figure 2a). Among the mutated genes, only MUC4 mutation $(P=0.002)$ was related to an inferior prognosis(Figure $2 \mathrm{~b})$. The results of univariate Cox model suggested that MUC4 mutation was correlated to a worsening of the outcome ( $\mathrm{HR}=2.084 \mathrm{p}=0.003,95 \% \mathrm{Cl}$ [1.286-3.376]) (Table 1). Afterward, the entire variables were analyzed via multivariate Cox regression. Further statistical tests revealed that the MUC4 mutation leads to an inferior prognosis ( $\mathrm{HR}=2.093, \mathrm{p}=0.00395 \% \mathrm{Cl}[1.290-3.396])$, suggesting that MUC4 mutation could act as an independent prognostic parameter in COAD patients.

Table 1

Exploration of the independent prognostic parameters in colon adenocarcinoma.

\begin{tabular}{|c|c|c|c|c|}
\hline \multirow[t]{2}{*}{ Factors } & \multicolumn{2}{|l|}{ Univariate } & \multicolumn{2}{|l|}{ Multivariate } \\
\hline & HR & $p$-value & HR & $p$-value \\
\hline age(year) $(<65, \geq 65)$ & 1.57ه0.99-2.50区 & 0.06 & & \\
\hline gender (male, female) & $1.37 \rrbracket 0.88-2.12 \rrbracket$ & 0.16 & & \\
\hline stage (low, high) & $2.82 \otimes 1.79-4.43 \rrbracket$ & 0.00 & $2.82 \rrbracket 1.79-4.43 \rrbracket$ & $<0.001$ \\
\hline MUC4 (wide, mutant) & 2.08ه1.29-3.38ه & $<0.001$ & $2.09 \rrbracket 1.29-3.40 \rrbracket$ & $<0.001$ \\
\hline
\end{tabular}




\section{Enrichment pathway analysis of MUC4 mutation}

We utilized GSEA enrichment analysis to reveal the underlying mechanism of MUC4 mutation邓the result showed that natural killer cell mediated cytotoxicity were mainly involved in patients with MUC4 mutation. Thus, we concluded that MUC4 mutation regulated pathways referred to the immune system (Figure 3).

\section{Correlation of MUC4 mutation with tumor microenvironment in COAD}

We further explored the connection between MUC4 mutation and tumor microenvironment in COAD patients. we compared the landscape of the immune cell in the MUC4 mutation group and wild group by CIBERSORT algorithm (Figure 4a). The difference analytical results showed that $\mathrm{CD} 8^{+} \mathrm{T}$ cells, activated NK cells and M1 macrophages were comparatively higher involved in tumors with MUC4 mutation. As shown in Figure $4 \mathrm{~b}$, resting memory $\mathrm{CD} 4^{+} \mathrm{T}$ cells were mainly enriched in the wild group. The outcome of Correlation analysis shown that $C D 8+T$ cells had a negative association with resting memory $C D 4^{+} T$ cells (Figure 4c).

\section{Discussion}

The tumorigenesis of colon adenocarcinoma is commonly known as a multi-stage process, which involves a series of genetic changes interacting with the tumor microenvironment $(T M E)[14,15]$. This study was conducted to analyze mutations in COAD samples from the TCGA dataset and ICGC dataset. We found a high mutation frequency of MUC4 in both cohorts. We were able to show that mutation in MUC4 was significantly correlated with increased TMB and indicated a worse prognosis. Moreover, samples with MUC4 mutations were enriched in the NK cell mediated cytotoxicity signaling pathway. The result of immune cells infiltration demonstrated that MUC4 mutated samples were more infiltrated in memory CD8 T cells, activated NK cells and M1 macrophages, but less infiltrated in resting memory CD4 T cells.

Some researches have revealed the relationship between tumor mutation burden (TMB) and immunotherapy response.MUC4 exhibited a relatively high mutation rate in COAD patients. Mucins are important glycoproteins, falling into two categories: the membrane-bound mucins and the secreted mucins[16, 17]. MUC4 is a useful biomarker of tumor progression[18, 19]. Its biological effects are mainly used in lung cancer, pancreatic cancer, ovarian and oesophageal malignancy [20-24]. MUC4 is expressed in epithelial cells of the digestive tract, as well as some goblet cells[25]. Abnormal MUC4 expression contributes to the progression of esophageal cancer[21] and colon cancer[26, 27]. Besides, an increased expression in MUC4 demonstrates a poor survival outcome, specifically in the early stages of colon cancer [28]. Due to the size of the MUC4 gene is relatively large, this may increase its mutation probability. Thus, MUC4 is the most common mutated gene in the state of stress exposure, for example, 
aging or nicotine treatment[29, 30]. MUC4 mutation was significant in Renal Carcinoma and was related to survival outcomes. The mutation of MUC4 could be tightly linked with smoking in kidney cancer [13] [31]. In present study, we revealed that COAD samples with a high rate of MUC4 mutation had a significantly worse survival outcome and were highly correlated with increased TMB. TMB has attracted attention as a biomarker for predicting effectiveness of immune checkpoint inhibitors, higher TMB may respond favorably to anti-PD-1/PD-L1 immunotherapy[32, 33]. However, recent studies [33, 34] confirmed that high TMB is a poor prognostic factor. The possible reasons are that high TMB tends to be associated with chromosomal instability that could depressed immune activities[35].

We used GSEA analysis to explore the potential biological function of MUC4 mutation in COAD. The result showed that the NK cell-mediated cytotoxicity pathway was the main driving mechanism for MUC4 mutation influencing the outcome for COAD patients. Together these functional analyses suggested that the MUC4 mutation had an important function in regulating the immune response in COAD.

Previous studies have indicated that the tumor immune microenvironment(TIM)has a significant meaning in predicting prognosis and evaluating therapeutic efficacy[8]. In our study, we found that that MUC4 mutation was significantly related to increased $C D 8^{+} T$ cells, NK cells and M1 macrophages. We hypothesized that a relatively higher proportion of $\mathrm{CD} 8^{+} \mathrm{T}$ cells in samples with MUC4 mutated was due to an increased number of tumor neoantigens, which strongly activated immune response. Interestingly, the correlation analysis result revealed that $\mathrm{CD} 8^{+} \mathrm{T}$ cells had a significant negative relation with resting memory $\mathrm{CD}^{+} \mathrm{T}$ cells. We also discovered a higher level of activated NK cells infiltrated in samples with MUC4 mutated, indicating that MUC4 mutation could stimulate activation of NK cells,however, more data are needed for various kinds of tumors. Studies show that NK cells were major players in the early detection, control of tumor metastasis in colon cancer[36-38]. Previous studies reported that M2-tumorassociated macrophages (M2-TAMs) were common in malignant tumors, which creates a favorable microenvironment for tumor progression[39, 40]. Meanwhile, M2-TAMs were found to be linked with poor survival outcome, while M1-TAMs point to a contrary conclusion [41-43]. M1 macrophages could be transformed into M2 with tumor progression. Interestingly, our study found that MUC4 mutated samples were more infiltrated in M1 macrophages. This is probably caused by an early staging of the majority cases in the TCGA datasets. Therefore, further studies are required to verify this hypothesis.

\section{Conclusions}

In summary, the present study implicates that MUC4 had a relatively high mutation rate in colon adenocarcinoma. MUC4 mutation was closely related to increased TMB and a worse prognosis. Finally, the NK cell mediated cytotoxicity signaling pathway were mainly enriched in samples with MUC4 mutations, and we also verified the association between mutations and immune cell infiltration. These findings identify that MUC4 may be considered a potential biomarker to predict the outcome of the immune response in colon adenocarcinoma. 


\section{Abbreviations}

COAD: colon adenocarcinoma; GSEA: Gene set enrichment analysis; ICGC: International Cancer Genome Consortium; M2-TAMs: M2-tumor-associated macrophages; MSI-H: high microsatellite instability; MUC4: Mucins 4; TCGA: The Cancer Genome Atlas; TIM: tumor immune microenvironment; TMB: tumor mutation burden; MMR: mismatch repair

\section{Declarations}

\section{Ethics approval and consent to participate}

Not applicable.

\section{Consent for publication}

Not applicable.

\section{Availability of Data and Materials}

The datasets analysed during the current study are available in the TCGA repository

(https://portal.gdc.cancer.gov/) and ICGC dataset (https://dcc.icgc.org/). The data is publicly available.

\section{Competing interests}

The authors declare that they have no competing interests.

\section{Funding}

Not applicable.

\section{Authors' contributions}

TL performed data analysis and wrote the manuscript. WJ.H collected the data. FG designed and revised the manuscript. The final manuscript was read and approved by all authors.

\section{Acknowledgements}

Not applicable. 


\section{References}

1. Network CGA: Comprehensive molecular characterization of human colon and rectal cancer. Nature, 2012; 487(7407):330-337

2. Manjelievskaia J, Brown D, Mcglynn KA, Anderson W, Shriver CD, Zhu K: Chemotherapy Use and Survival Among Young and Middle-Aged Patients With Colon Cancer. Jama Surg, 2017; 152(5):452459

3. Al Bandar MH, Kim NK: Current status and future perspectives on treatment of liver metastasis in colorectal cancer (Review). Oncol Rep, 2017; 37(5):2553-2564

4. Goodman AM, Kato S, Bazhenova L et al: Tumor Mutational Burden as an Independent Predictor of Response to Immunotherapy in Diverse Cancers. Mol Cancer Ther, 2017; 16(11):2598-2608

5. Hellmann MD, Ciuleanu TE, Pluzanski A et al: Nivolumab plus Ipilimumab in Lung Cancer with a High Tumor Mutational Burden. N Engl J Med, 2018; 378(22):2093-2104

6. Matsushita H, Vesely MD, Koboldt DC et al: Cancer exome analysis reveals a T-cell-dependent mechanism of cancer immunoediting. Nature, 2012; 482(7385):400-404

7. Riaz N, Morris L, Havel JJ, Makarov V, Desrichard A, Chan TA: The role of neoantigens in response to immune checkpoint blockade. Int Immunol, 2016; 28(8):411-419

8. Cao D, Xu H, Xu X, Guo T, Ge W: High tumor mutation burden predicts better efficacy of immunotherapy: a pooled analysis of 103078 cancer patients. Oncoimmunology, 2019; 8(9):e1629258

9. Schrock AB, Ouyang C, Sandhu J et al: Tumor mutational burden is predictive of response to immune checkpoint inhibitors in MSI-high metastatic colorectal cancer. Ann Oncol, 2019; 30(7):1096-1103

10. Le DT, Uram JN, Wang H et al: PD-1 Blockade in Tumors with Mismatch-Repair Deficiency. N Engl J Med, 2015; 372(26):2509-2520

11. Fabrizio DA, Jr TJG, Dunne RF, Frampton G, Klempner SJ: Beyond microsatellite testing: assessment of tumor mutational burden identifies subsets of colorectal cancer who may respond to immune checkpoint inhibition. Journal of Gastrointestinal Oncology, 2018; 9(4):610-617

12. Stadler ZK, Battaglin F, Middha S et al: Reliable Detection of Mismatch Repair Deficiency in Colorectal Cancers Using Mutational Load in Next-Generation Sequencing Panels. J Clin Oncol, 2016; 34(18):2141-2147

13. Hunt JD, Van Der Hel OL, Mcmillan GP, Boffetta P, Brennan P: Renal cell carcinoma in relation to cigarette smoking: meta-analysis of 24 studies. Int J Cancer, 2005; 114(1):101-108

14. Zeng Q, Zhang W, Li X, Lai J, Li Z: Bioinformatic identification of renal cell carcinoma microenvironment-associated biomarkers with therapeutic and prognostic value. Life Sci, 2020; 243:117273

15. Hsu YC, Chang YH, Chang GC et al: Tumor mutation burden and recurrent tumors in hereditary lung cancer. 2019; 8(5):2179-2187 
16. Corfield AP: Mucins: a biologically relevant glycan barrier in mucosal protection. Biochim Biophys Acta, 2015; 1850(1):236-252

17. Dekker J, Rossen JW, Büller HA, Einerhand AW: The MUC family: an obituary. Trends Biochem Sci, 2002; 27(3):126-131

18. Jonckheere N, Van Seuningen I: Integrative analysis of the cancer genome atlas and cancer cell lines encyclopedia large-scale genomic databases: MUC4/MUC16/MUC20 signature is associated with poor survival in human carcinomas. J Transl Med, 2018; 16(1):259

19. Mercogliano MF, De Martino M, Venturutti L et al: TNFa-Induced Mucin 4 Expression Elicits Trastuzumab Resistance in HER2-Positive Breast Cancer. Clin Cancer Res, 2017; 23(3):636-648

20. Jonckheere N, Skrypek N, Merlin J et al: The mucin MUC4 and its membrane partner ErbB2 regulate biological properties of human CAPAN-2 pancreatic cancer cells via different signalling pathways. Plos One, 2012; 7(2):e32232

21. Emilie, Bruyère, And et al: The MUC4 membrane-bound mucin regulates esophageal cancer cell proliferation and migration properties: Implication for S100A4 protein. Biochemical \& Biophysical Research Communications, 2011; 413(2):325-329

22. Skrypek N, Duchêne B, Hebbar M, Leteurtre E, Van Seuningen I, Jonckheere N: The MUC4 mucin mediates gemcitabine resistance of human pancreatic cancer cells via the Concentrative Nucleoside Transporter family. Oncogene, 2013; 32(13):1714-1723

23. Bafna S, Kaur S, Momi N, Batra SK: Pancreatic cancer cells resistance to gemcitabine: the role of MUC4 mucin. Br J Cancer, 2009; 101(7):1155-1161

24. Kaur S, Kumar S, Momi N, Sasson AR, Batra SK: Mucins in pancreatic cancer and its microenvironment. Nat Rev Gastroenterol Hepatol, 2013; 10(10):607-620

25. Andrianifahanana M, Moniaux N, Batra SK: Regulation of mucin expression: mechanistic aspects and implications for cancer and inflammatory diseases. Biochim Biophys Acta, 2006; 1765(2):189222

26. Biemer-Hüttmann AE, Walsh MD, Mcguckin MA et al: Mucin core protein expression in colorectal cancers with high levels of microsatellite instability indicates a novel pathway of morphogenesis. Clin Cancer Res, 2000; 6(5):1909-1916

27. Ogata $\mathrm{S}$, Uehara $\mathrm{H}$, Chen A, Itzkowitz SH: Mucin gene expression in colonic tissues and cell lines. Cancer Res, 1992; 52(21):5971-5978

28. Shanmugam C, Jhala NC, Katkoori VR et al: Prognostic value of mucin 4 expression in colorectal adenocarcinomas. Cancer, 2010; 116(15):3577-3586

29. Bavarva JH, Tae H, Mciver L, Garner HR: Nicotine and oxidative stress induced exomic variations are concordant and overrepresented in cancer-associated genes. Oncotarget, 2014; 5(13):4788-4798

30. Bavarva JH, Tae H, Mciver L, Karunasena E, Garner HR: The dynamic exome: acquired variants as individuals age. Aging (Albany NY), 2014; 6(6):511-521 
31. Hayat MJ, Howlader N, Reichman ME, Edwards BK: Cancer statistics, trends, and multiple primary cancer analyses from the Surveillance, Epidemiology, and End Results (SEER) Program. Oncologist, $2007 ; 12(1): 20-37$

32. Innocenti F, Ou FS, Qu X et al: Mutational Analysis of Patients With Colorectal Cancer in CALGB/SWOG 80405 Identifies New Roles of Microsatellite Instability and Tumor Mutational Burden for Patient Outcome. Journal of Clinical Oncology, 2019; 37(14):1217-1227

33. Wang $\mathrm{X}$, Li M: Correlate tumor mutation burden with immune signatures in human cancers. BMC Immunol, 2019; 20(1):4

34. Owada-Ozaki Y, Muto S, Takagi H et al: Prognostic Impact of Tumor Mutation Burden in Patients With Completely Resected Non-Small Cell Lung Cancer: Brief Report. J Thorac Oncol, 2018; 13(8):1217-1221

35. Jiang Z, Liu Z, Li M, Chen C, Wang X: Immunogenomics Analysis Reveals that TP53 Mutations Inhibit Tumor Immunity in Gastric Cancer. Transl Oncol, 2018; 11(5):1171-1187

36. Kondo E, Koda K, Takiguchi $\mathrm{N}$ et al: Preoperative natural killer cell activity as a prognostic factor for distant metastasis following surgery for colon cancer. Dig Surg, 2003; 20(5):445-451

37. Cui F, Qu D: The Role of Circulating CD16 + CD56 + Natural Killer Cells in the Screening, Diagnosis, and Staging of Colorectal Cancer before Initial Treatment. Dis Markers, 2019; 2019:7152183

38. Spacek J, Vocka M: Immunological examination of peripheral blood in patients with colorectal cancer compared to healthy controls. Immunol Invest, 2018; 47(7):643-653

39. Hao NB, Lü MH, Fan YH, Cao YL, Zhang ZR, Yang SM: Macrophages in tumor microenvironments and the progression of tumors. Clin Dev Immunol, 2012; 2012:948098

40. Lewis CE, Pollard JW: Distinct role of macrophages in different tumor microenvironments. Cancer Res, 2006; 66(2):605-612

41. Kawachi A, Yoshida H, Kitano S, Ino Y, Kato T, Hiraoka N: Tumor-associated CD204(+) M2 macrophages are unfavorable prognostic indicators in uterine cervical adenocarcinoma. Cancer Sci, 2018; 109(3):863-870

42. Kim, Younghoon, Wen et al: The distribution of intratumoral macrophages correlates with molecular phenotypes and impacts prognosis in colorectal carcinoma. Histopathology Official Journal of the British Division of the International Academy of Pathology, 2018; 73(4):663-671

43. Yuan X, Zhang J, Li D et al: Prognostic significance of tumor-associated macrophages in ovarian cancer: A meta-analysis. Gynecol Oncol, 2017; 147(1):181-187

\section{Figures}



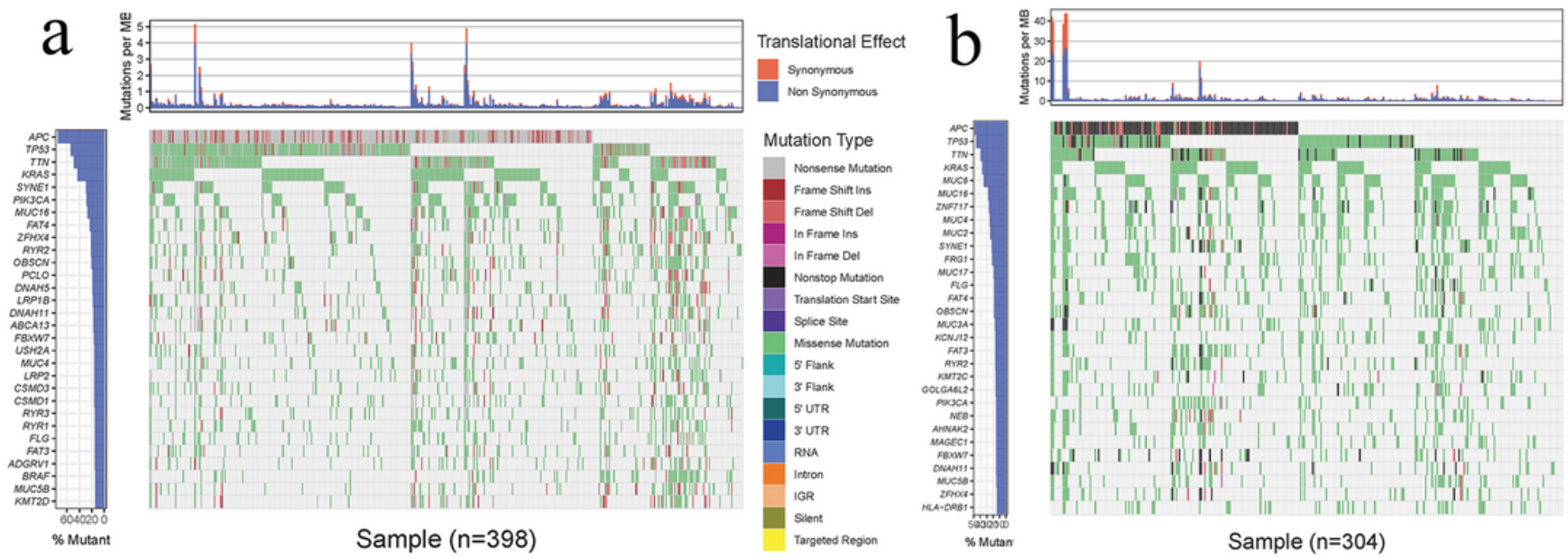

Translational Effect
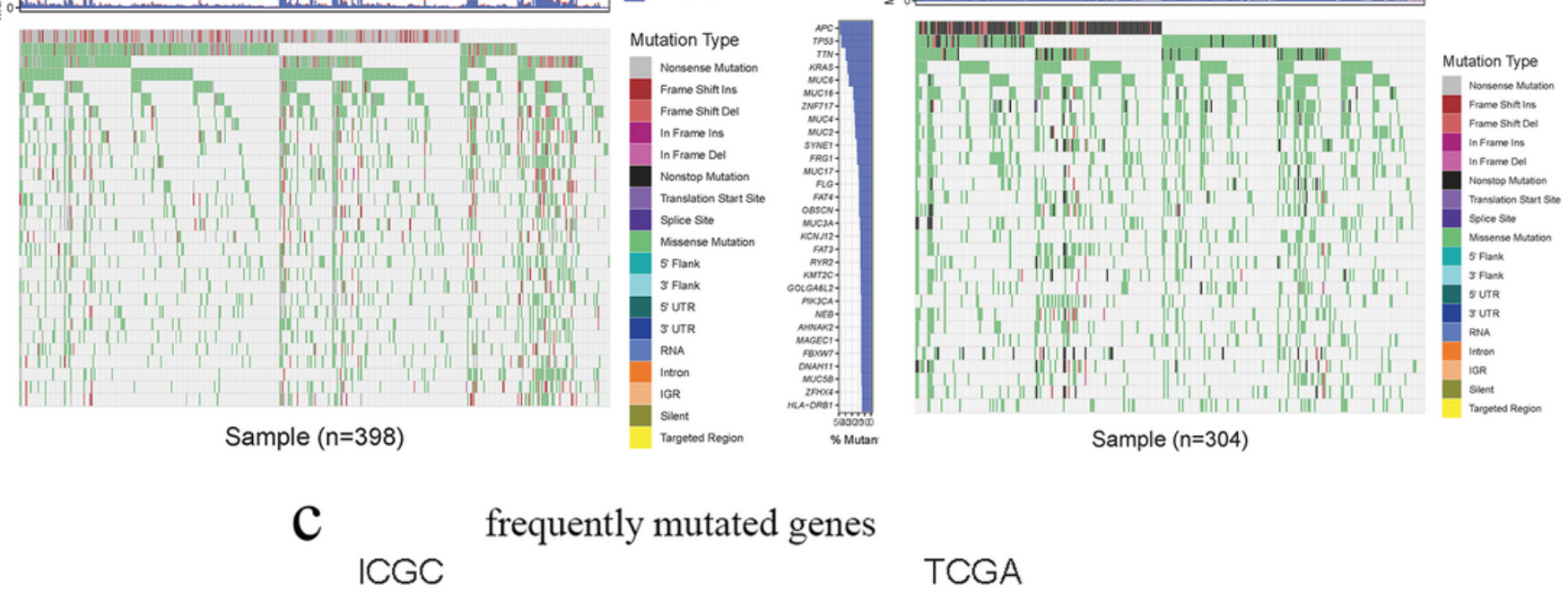

TCGA

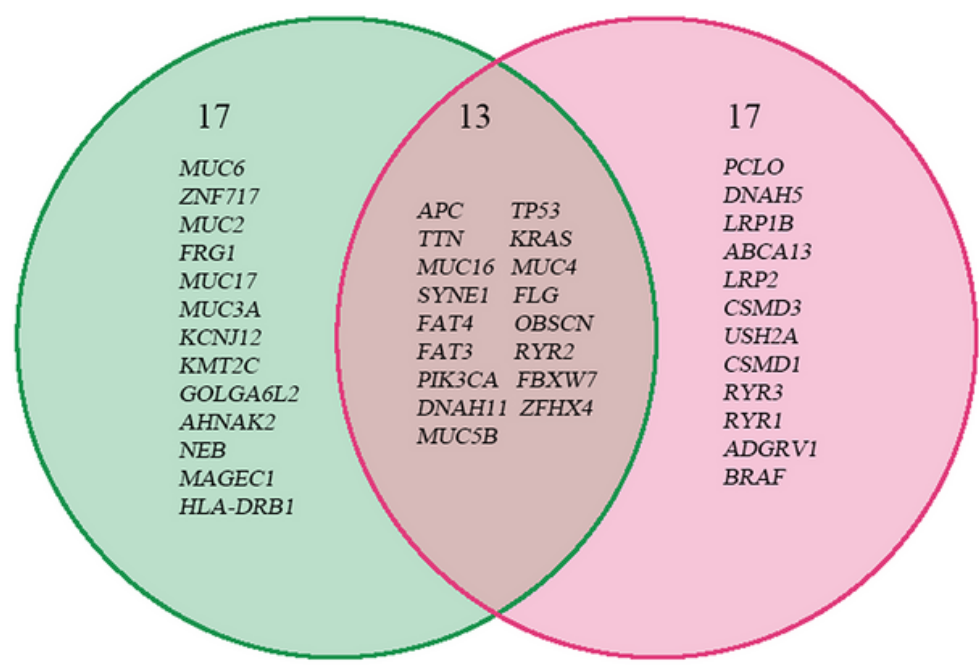

Figure 1

Primary mutation of genes in colon adenocarcinoma. (a) Waterfall of the top 30 mutated genes in the COAD patients from the TCGA cohort. (b) Waterfall of the top 30 mutated genes in the COAD patients from the ICGC cohort. (c) Venn diagram of the top 30 mutated genes shared by two cohorts. 


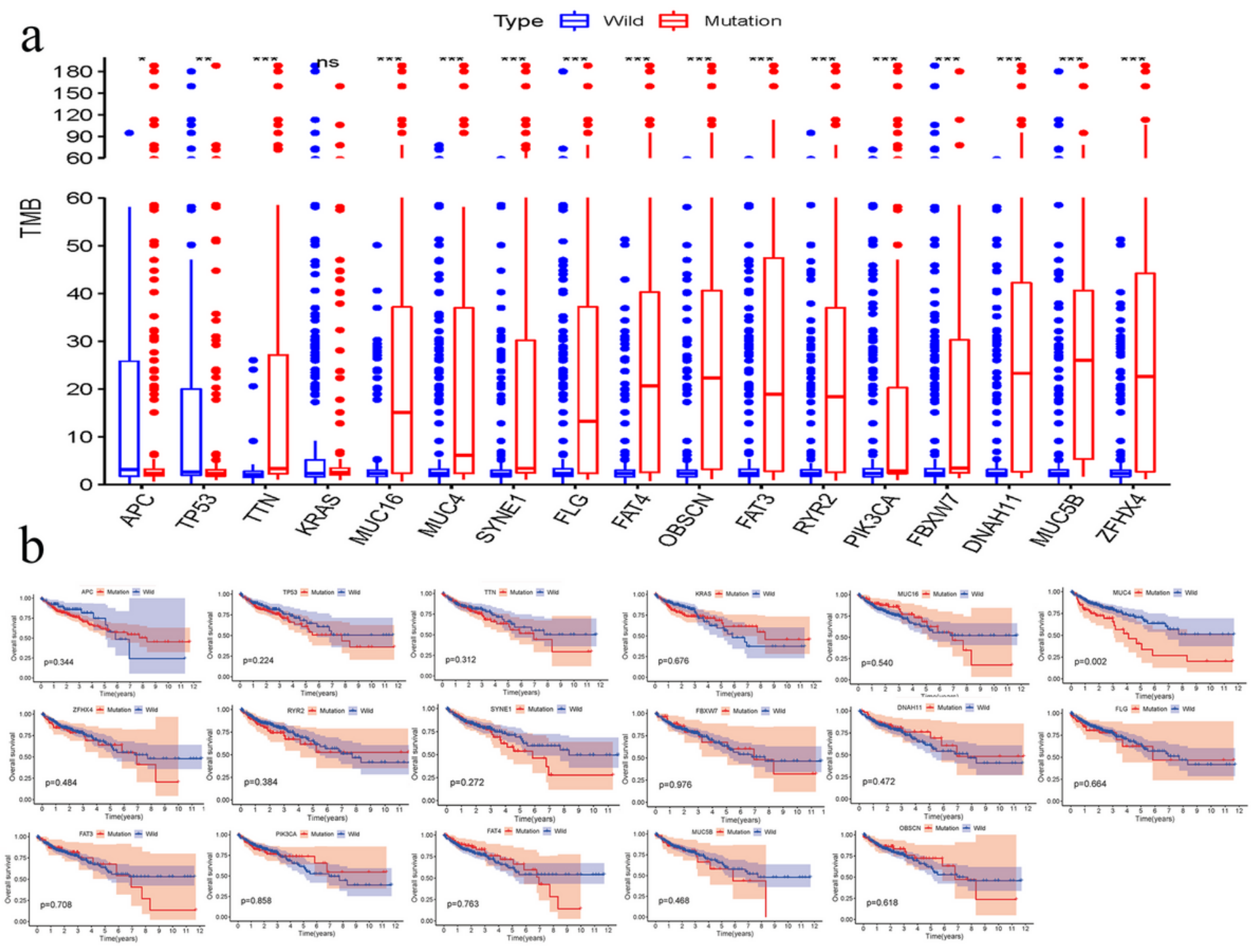

Figure 2

Correlation of mutated Gene with TMB and prognosis of COAD patients. (a) The majority of mutated genes are highly associated with higher TMB. ${ }^{*} p<0.05 * * p<0.01$; *** $p<0.001$. (b) $\mathrm{K}-\mathrm{M}$ curves of patients with gene mutations. WT, wild type; MT, mutant type. 


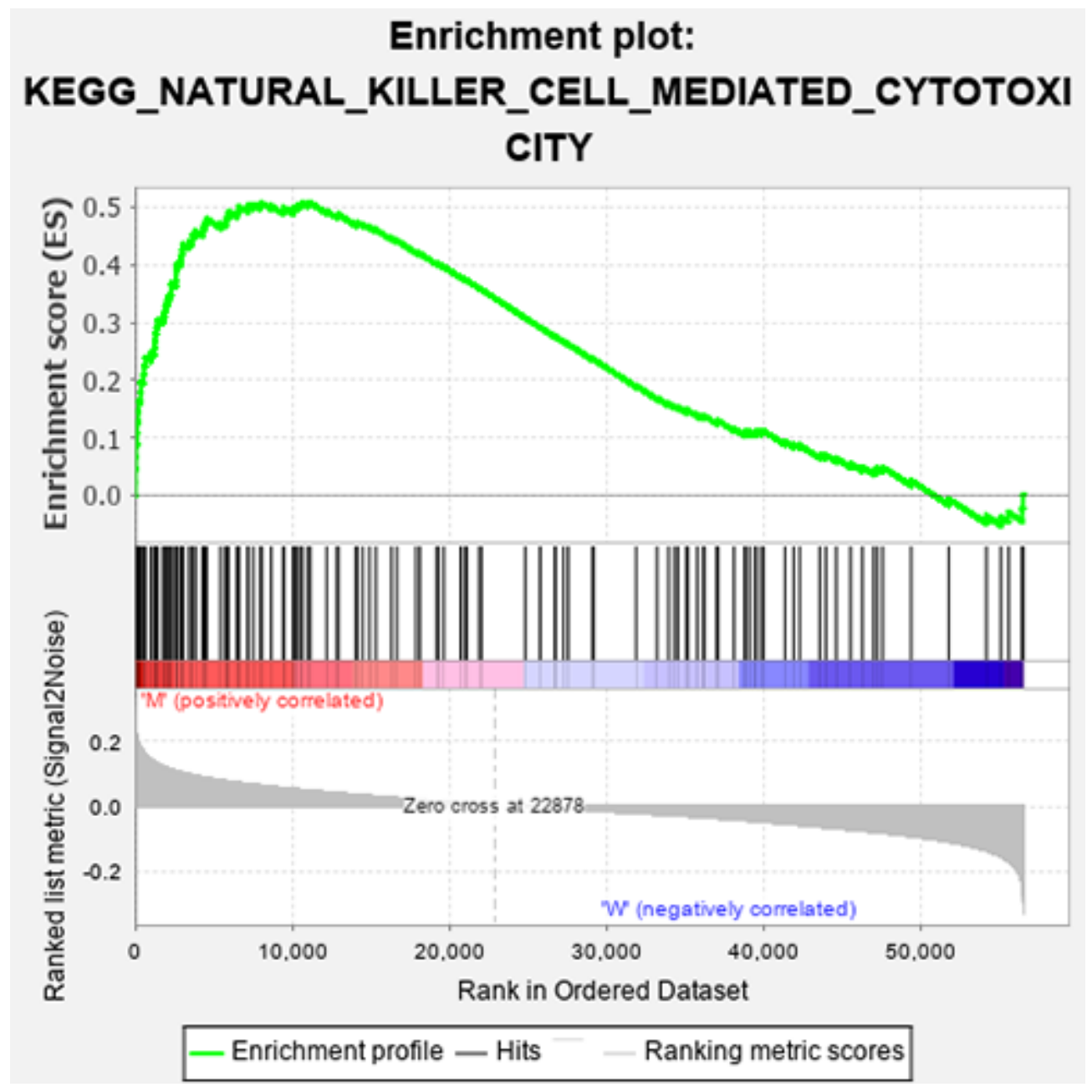

Figure 3

MUC4 mutation correlated enrichment gene analysis with GSEA. NK cell-mediated cytotoxicity was mainly involved in samples with MUC4 mutation. 


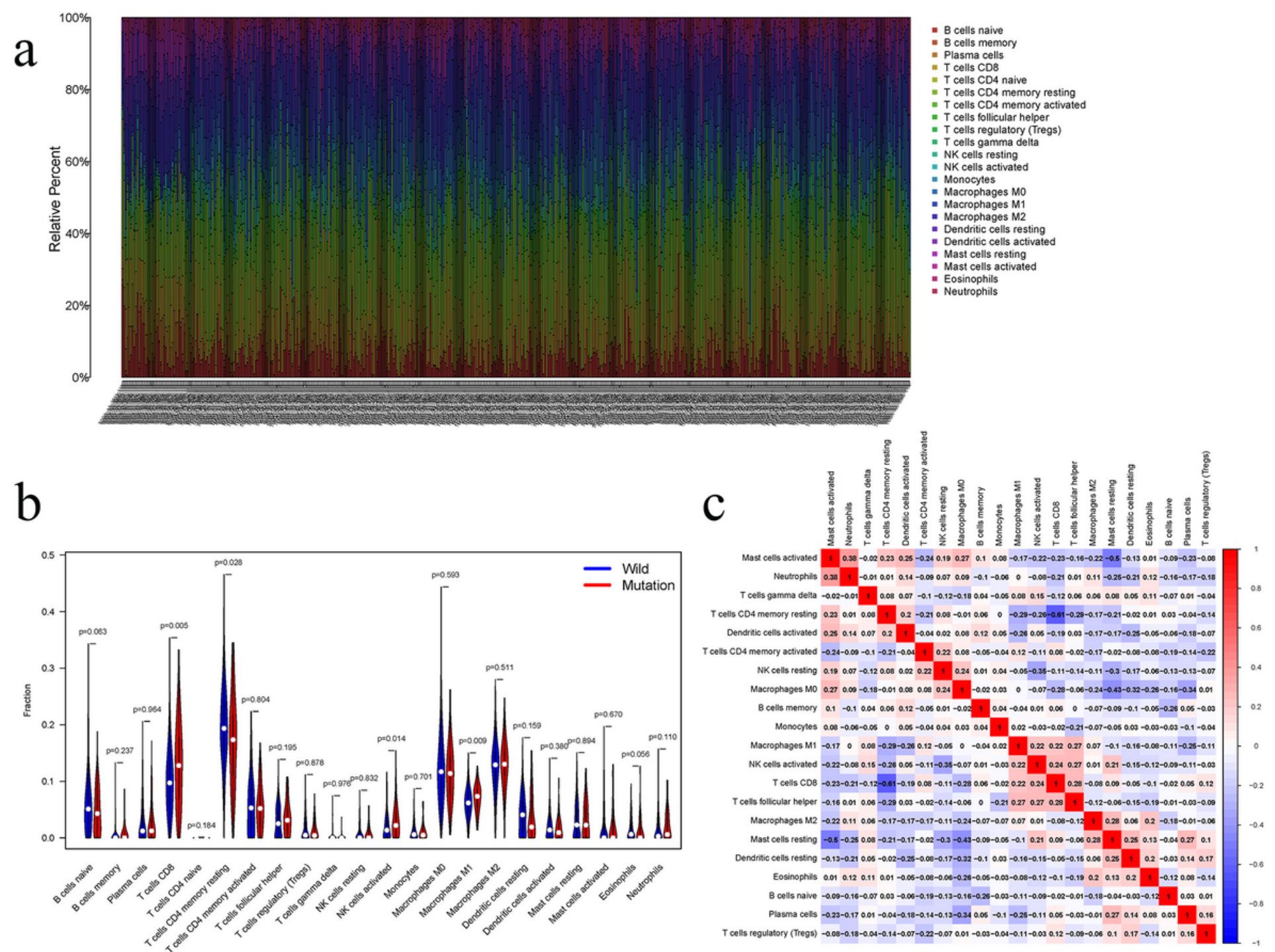

\section{Figure 4}

MUC4 mutation is highly related to tumor microenvironment. (a) The landscape of tumor-infiltrating immune cells in TCGA samples. (b) The proportions of infiltrated immune cells in MUC4 mutation groups and MUC4 wild group. (c) correlation analysis of the 22 immune cells. 